\title{
Fatores associados à obesidade infantil: uma revisão de literatura
}

\author{
Factors associated with childhood obesity: an literature review
}

Factores asociados con la obesidad infantil: una revisión literatura

\author{
Bruna Rocha Ferreira1', Ester de Melo Costa ${ }^{1}$, Maria Eduarda Rocha Machado Fonseca1*, Gérsika \\ Bitencourt Santos ${ }^{1}$.
}

\section{RESUMO}

Objetivo: Realizar uma revisão bibliográfica, com enfoque na obesidade infantil e discutir os fatores que influenciam no estilo de vida das crianças, como a mídia audiovisual, as relações no núcleo familiar, as escolas e a ausência de atividade física. Métodos: Os artigos foram selecionados pelas bases de dados PubMed, Scielo, LILACS e Google Acadêmico e publicados entre os anos 2004 a 2019, utilizando os descritores "obesidade infantil", "família", "escola". Resultados: Foram encontrados 315 artigos, selecionados os publicados entre 2010 a 2020, e utilizados 20 artigos para comporem esta revisão de literatura. Dentre os 20 artigos selecionados e analisados todos representaram o cenário brasileiro (artigos nacionais). Considerações finais: O ambiente onde a criança está inserida, tanto o familiar, o escolar quanto o social, é um componente facilitador para manifestação de obesidade na infância, que por sua vez influencia no desenvolvimento de diversas patologias na vida adulta. Logo, se faz necessário a mudança de hábitos sedentários e patogênicos, a partir de uma psicoeducação.

Palavras-chave: Obesidade infantil, Fatores de risco, Complicações.

\begin{abstract}
Objective: To carry out a bibliographic review, focusing on childhood obesity and discuss the factors that influence children's lifestyle, such as audiovisual media, family relationships, schools and lack of physical activity. Methods: The articles were selected by the PubMed, Scielo, LILACS and Google Scholar databases and published between the years 2004 to 2019, using the descriptors "childhood obesity", "family", "school". Results: We found 315 articles, selected those published between 2010 and 2020, and 20 articles were used to compose this literature review. Among the 20 articles selected and analyzed, all represented the Brazilian scenario (national articles). Final considerations: The context in which the child is inserted, whether family, school or social, can promote a facilitating combination for the emergence of childhood obesity, which in turn contributes to the emergence of pathologies in adulthood. Therefore, changes in sedentary and pathogenic habits are necessary, based on psychoeducation.
\end{abstract}

Key words: Childhood obesity, Risk factors, Complications.

\section{RESUMEN}

Objetivo: Realizar una revisión bibliográfica, centrándose en la obesidad infantil y discutir los factores que influyen en el estilo de vida de los niños, como los medios audiovisuales, las relaciones familiares, las escuelas y la falta de actividad física. Métodos: Los artículos fueron seleccionados por las bases de datos PubMed, Scielo, LILACS y Google Scholar y publicados entre los años 2010 a 2020, utilizando los descriptores "obesidad infantil", "familia", "escuela". Resultados: Se encontraron un total de 315 artículos, seleccionados fueron los publicados entre 2004 y 2020, y se utilizaron 20 artículos para componer esta revisión de la literatura. Entre los 20 artículos seleccionados y analizados, todos representaron el escenario brasileño

${ }^{1}$ Universidade José do Rosário Vellano (UNIFENAS), Alfenas - MG.

*E-mail: Mariaeduardafonseca0302@gmail.com

SUBMETIDO EM: 4/2021

ACEITO EM: 5/2021

PUBLICADO EM: 5/2021 
(artículos nacionales). Consideraciones finales: El contexto en el que se inserta el niño, ya sea familiar, escolar o social, puede promover una combinación facilitadora para la aparición de la obesidad infantil, lo que a su vez contribuye a la aparición de patologías en la edad adulta. Por tanto, son necesarios cambios en los hábitos sedentarios y patógenos, basados en la psicoeducación.

Palabras-clave: Obesidad infantil, Factores de riesgo, Complicaciones.

\section{INTRODUÇÃO}

A obesidade é avaliada como uma patologia crônica não transmissível, que se caracteriza por apresentar alto grau de armazenamento de gordura corporal e ausência de dor física. Essa patologia apresenta as seguintes propriedades: latência prolongada, largo período assintomático, curso clínico vagaroso, manifestações clínicas permanentes com diferentes etapas de exacerbação (LOPES PCS, et al., 2010).

Com o crescente número de casos em escala mundial, a obesidade na infância é um assunto em ascensão que causa grande preocupação (LINHARES FMM, et al., 2016). A Comissão de Obesidade no Brasil (COB) revelou que o sobrepeso e a obesidade são encontrados com grande frequência, a partir dos 05 anos de idade, em todos os grupos de renda e em todas as regiões brasileiras (SANTOS LRC e RABINOVICH EP, 2011).

Segundo último relatório sobre a obesidade infantil liberado pela Organização Mundial da Saúde (OMS), aproximadamente 41 milhões de crianças menores de 5 anos estavam obesas ou acima do peso em 2014 (LIRA AK, et al., 2020).

A obesidade pode ter início em qualquer idade e seu desenvolvimento está relacionado a fatores genéticos, metabólicos e fisiológicos, os quais podem ser agravados pelo ambiente. Dentre os fatores ambientais que podem levar a obesidade infantil, pode-se citar: a influência midiática, que estimula uma alimentação inadequada ao mesmo tempo que cobra um padrão ideal de magreza; e a economia/política que estimula tanto o consumismo quanto os interesses da indústria da alimentação não saudável (SANTOS LRC e RABINOVICH EP, 2011; LOPES PCS, et al., 2010).

Ademais, a obesidade pode ser desencadeada, ainda, por outros fatores como: introdução inadequada de alimentos, desmame precoce, distúrbios no comportamento alimentar e complicações no ambiente familiar (LOPES PCS, et al., 2010).

Segundo, Galindo DS e Assolini PJ (2008), as crianças têm demonstrado poder de fisgar e convencer os pais de que precisam de certos produtos. Especificamente com a alimentação, os autores usam o termo "entertainment", utilizado pela indústria de alimento para se referir a associação entre comida e diversão, estratégia muito aplicada em brindes que vem com os alimentos, criando também sentidos e emoções no público infantil. Eles afirmam que essa tática potencializa ainda mais o consumo das crianças, o que gera ao mercado lucros exorbitantes.

Uma das muitas consequências causadas pela obesidade na infância é o surgimento de psicopatologias. Na pesquisa realizada por Bertoletti J e Santos SCG (2012), mais da metade das crianças apresentavam níveis de estresse, e uma pequena quantidade já fazia uso de medicamentos psiquiátricos. Ademais, a obesidade tem poder desfavorável sobre quadros de ansiedade, autoestima e depressão (MENDES JOH, et al., 2019).

Além de distúrbios psicológicos, a obesidade está altamente relacionada a diferentes comorbidades que virão a aparecer na vida adulta, como hipertensão arterial, diabetes mellitus tipo II, doenças cardíacas, osteoartrite, entre outros (LOPES PCS, et al., 2010).

Portanto, este artigo objetivou realizar uma revisão bibliográfica, com enfoque na obesidade infantil e discutir os fatores que têm influência nos hábitos de vida das crianças, como a mídia audiovisual, as relações no núcleo familiar, as escolas e a ausência de atividade física. 


\section{MÉTODOS}

Trata-se de uma revisão integrativa de literatura. A pesquisa ocorreu nas bases de dados Scientific Electronic Library Online (SCIELO), Literatura Latino-Americana e do Caribe em ciências da saúde (LILACS), PubMed e Google Acadêmico. Para tanto foi utilizado critério de inclusão de artigos publicados entre os anos de 2010 a 2020. Foram utilizados como descritores: "obesidade infantil, comportamento e hábitos alimentares, influência da família, escola e mídia". Após a busca deste material foram encontrados 315 artigos os quais continham as palavras-chave de nosso estudo, sendo selecionados 20 artigos para compor a amostra (Figura 1).

A coleta de dados iniciou-se mediante a pré-seleção dos artigos, conforme os critérios de inclusão. Após isso, foram selecionados os artigos que tratavam do tema de interesse. Os dados analisados após leitura dos artigos selecionados, mostraram temas específicos como: má alimentação, ausência de atividade física e influência genética, psicologia associada à obesidade, fatores familiares e obesidade, influências da mídia.

Figura 1 - Distribuição das referências obtidas nas 4 bases de dados utilizadas para realização do estudo, segundo as palavras chaves selecionadas.

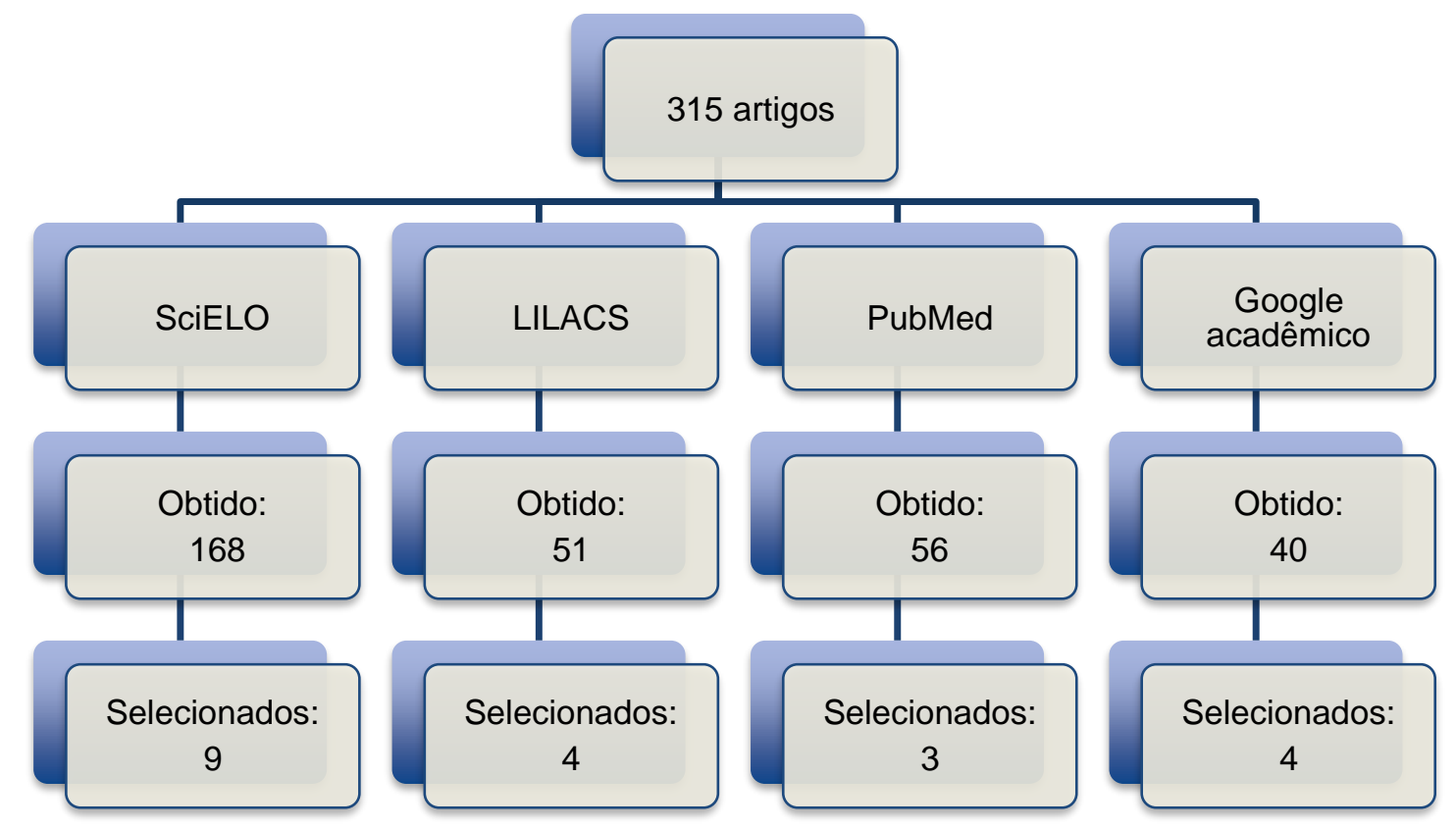

Fonte: Ferreira BR, et al., 2021.

\section{RESULTADOS}

Após a busca nas bases de dados sobre o tema obesidade infantil, foram encontrados 315 artigos, selecionados os publicados entre 2010 a 2020, e utilizados 20 artigos para comporem esta revisão de literatura. Dentre os 20 artigos selecionados e analisados, publicados entre os anos de 2010 e 2020, todos representaram o cenário brasileiro (artigos nacionais). Os artigos selecionados estão representados no

\section{Quadro 1.}

Dos 20 artigos escolhidos para revisão 5 tinham foco na prevenção da obesidade, 5 traziam a relação da obesidade com aspectos psicológicos, 4 relacionavam a obesidade com o âmbito familiar, 3 eram estudos analíticos quantitativos, 2 relacionavam a obesidade com outros fatores como dislipidemia e COVID-19 (Coronavírus Disease), enquanto 1 refletia sobre a composição corporal. 
Quadro 1 - Síntese dos principais achados sobre obesidade infantil dentre os 20 artigos escolhidos para a revisão.

\begin{tabular}{|c|c|c|c|}
\hline Ano & Autores & Revista & Principais achados \\
\hline 2010 & Lopes PCS, et al. & Rev. bras. enferm & $\begin{array}{l}\text { O artigo de revisão tem como enfoque a influência dos pais no desenvolvimento da obesidade infantil, } \\
\text { principalmente quando se fala em hábitos alimentares. Analisa que os pais, como provedores de } \\
\text { soluções devem disseminar boas práticas alimentares e hábitos de vidas mais saudáveis. }\end{array}$ \\
\hline 2010 & Tassara V, et al. & Rev. paul. pediatr & $\begin{array}{l}\text { Estudo descritivo qualitativo que traz a compreensão das relações vividas por crianças obesas no } \\
\text { âmbito familiar e social. Analisa a complexidade da obesidade infantil e os fatores que se relacionam } \\
\text { para que a mesma ocorra. }\end{array}$ \\
\hline 2011 & $\begin{array}{l}\text { Santos LRC e } \\
\text { Rabinovich EP }\end{array}$ & Saude soc. & $\begin{array}{l}\text { O estudo analítico qualitativo tem como objetivo analisar a relação entre filhos únicos e obesidade. } \\
\text { O ensaio também discuti outros assuntos como: o crescente índice de obesidade no Brasil; a } \\
\text { inserção da mulher no mercado de trabalho como causadora das mudanças no dinamismo familiar; }\end{array}$ \\
\hline 2012 & $\begin{array}{c}\text { Bertoletti J e Santos } \\
\text { SCG }\end{array}$ & PSICO & $\begin{array}{l}\text { O estudo descritivo quantitativo tem ênfase no estresse como papel de relevância na gênese da } \\
\text { obesidade. O mesmo discute também outros assuntos relacionados, como a ascensão da ansiedade } \\
\text { na sociedade moderna e sua prevalência quando se trata de obesidade infantil. }\end{array}$ \\
\hline 2013 & Camargo APPM, et al. & Ciênc. saúde coletiva & $\begin{array}{l}\text { O artigo de revisão tem assunto central no papel da família no tratamento da obesidade infantil. } \\
\text { Apesar do seu tema central, o artigo também gira em torno de assuntos relacionados, como: os } \\
\text { efeitos da globalização como explicação para mudança nos hábitos alimentares atuais; }\end{array}$ \\
\hline 2014 & Dornelles AD, et al. & Saúde soc. & $\begin{array}{l}\text { O estudo descritivo qualitativo tem como tema central a visão dos profissionais de saúde acerca do } \\
\text { seguinte tema: a importância de intervir nos hábitos de vida dos familiares para um melhor tratamento } \\
\text { da obesidade infantil. }\end{array}$ \\
\hline 2014 & Andrade TM, et al. & Psicol. cienc. prof & $\begin{array}{l}\text { O artigo de revisão descreve sobre os principais problemas psicológicos enfrentados por crianças e } \\
\text { adolescentes obesos. Apresenta também destaque para obesidade como consequência de } \\
\text { interações familiares conflituosas: superproteção, rejeição materna, maus tratos, entre outros. }\end{array}$ \\
\hline 2015 & Miranda JMQ, et al. & Rev Bras Med Esporte & $\begin{array}{l}\text { O estudo analítico quantitativo compara a prevalência do sobrepeso e obesidade em estudantes da } \\
\text { rede pública e privada, sendo relatado maior índice de obesidade em estudantes da rede privada. } \\
\text { Estudo realizado a partir de análise das variáveis antropométricas nos sexos feminino e masculino. }\end{array}$ \\
\hline 2016 & Linhares FMM, et al. & Temas em Saúde & $\begin{array}{l}\text { A revisão integrativa da literatura apresenta a influência familiar nos hábitos alimentares e no estilo } \\
\text { de vida dos filhos, que podem predispor a uma futura obesidade. Os autores levaram em } \\
\text { consideração fatores como: consumo, cultura, relacionamentos entre outros. }\end{array}$ \\
\hline 2017 & Oliveira LC, et al. & Rev Saúde Pública & $\begin{array}{l}\text { O estudo analítico quantitativo discorre sobre a importância da realização de atividades físicas com } \\
\text { acelerômetro. Ressaltando-se as recomendações necessárias de passos/dia e/ou min/dia de } \\
\text { Atividade Física de Moderada a Vigorosa (AFMV), para prevenir a obesidade infantil. }\end{array}$ \\
\hline
\end{tabular}




\begin{tabular}{|c|c|c|c|}
\hline Ano & Autores & Revista & $\begin{array}{ll}\text { Principais achados } \\
\end{array}$ \\
\hline 2017 & Testa WL, et al. & RBONE & $\begin{array}{l}\text { O ensaio clínico controlado tem por objetivo investigar a importância da realização de exercícios } \\
\text { físicos e de acompanhamento nutricional na intervenção da obesidade infantil. Ressalta-se também } \\
\text { a relevância de atividades recreativas no lugar de atividades físicas estruturadas. }\end{array}$ \\
\hline 2018 & Andrade RS, et al. & RBAC & $\begin{array}{l}\text { A revisão literária sistemática tem como enfoque a correlação entre obesidade e dislipidemia, } \\
\text { comparando seus índices. A revisão também evidencia a relação entre dislipidemia e seus } \\
\text { marcadores laboratoriais (HDL, LDL e VLDL). }\end{array}$ \\
\hline 2018 & Henriques $\mathrm{P}$, et al. & Ciênc. saúde coletiva & $\begin{array}{l}\text { O artigo de revisão teve como objetivo analisar as ações relacionadas a prevenção e controle da } \\
\text { obesidade, a partir de documentos que integram as Políticas do governo Brasileiro. }\end{array}$ \\
\hline 2018 & Paiva ACT, et al. & Revista Cuidarte & $\begin{array}{l}\text { Estudo analítico quantitativo que objetivou encontrar alterações em escolares. O Estudo também } \\
\text { destaca os fatores de risco para obesidade, ressaltando o estilo de vida moderno como principal } \\
\text { agravante. Aponta-se também o papel da família no estilo de vida que será adotado pela criança no } \\
\text { futuro. }\end{array}$ \\
\hline 2018 & Perez LM e Mattiello $R$ & Revista Cuidarte. & $\begin{array}{l}\text { O artigo de reflexão disserta acerca da definição de composição corporal. Cita-se também os } \\
\text { diferentes tipos de ferramentas utilizados para avaliação do estado nutricional. Além de discorrer } \\
\text { sobre a importância de se considerar outros fatores na composição corporal além do IMC. }\end{array}$ \\
\hline 2019 & Mendes $\mathrm{JOH}$, et al. & Rev. SBPH & $\begin{array}{l}\text { O artigo de revisão tem como propósito analisar os sintomas de depressão e ansiedade em crianças } \\
\text { obesas. Além de discutir outros assuntos, como: o vínculo entre as trocas afetivas dentro ambiente } \\
\text { familiar com a obesidade infantil. }\end{array}$ \\
\hline 2019 & Almeida NB, et al. & Rev. paul. pediatr & $\begin{array}{l}\text { O artigo descritivo quantitativo tem como intuito identificar os fatores responsáveis pelo ganho } \\
\text { ponderal excessivo entre pré-escolares de Centros de Educação Infantil, com foco principal no } \\
\text { aleitamento materno exclusivo. }\end{array}$ \\
\hline 2020 & Costa LR, et al. & Residência Pediátrica & $\begin{array}{l}\text { O artigo de revisão de literatura tem como objetivo correlacionar a obesidade infantil com casos } \\
\text { graves de COVID-19. Traz uma breve explicação do que é a obesidade e explica como o seu estado } \\
\text { inflamatório crônico no organismo leva a uma piora nos quadros de infecção por SARS-CoV-2. }\end{array}$ \\
\hline 2020 & Coutinho VM, et al. & Rev. paul. pediatr & $\begin{array}{l}\text { O artigo de revisão de literatura tem como foco o estilo de apego desenvolvido por crianças com } \\
\text { doenças crônicas. Tendo enfoque em estratégias alternativas para prevenção da obesidade, como } \\
\text { auxiliar na regulação das emoções. }\end{array}$ \\
\hline 2020 & Lira AK, et al. & Temas em Saúde & $\begin{array}{l}\text { O artigo de revisão tem enfoque na identificação dos fatores que determinam o sobrepeso e a } \\
\text { obesidade infantil. Analisando a ingestão exagerada de alimentos industrializados e a falta de } \\
\text { atividade física como principais causadores. }\end{array}$ \\
\hline
\end{tabular}

Fonte: Ferreira BR, et al., 2021. 


\section{DISCUSSÃO}

É consenso entre os autores dos 20 artigos selecionados para esse estudo, que a obesidade é uma patologia classificada como multifatorial, abrangendo fatores genéticos, sociais, econômicos, psicológicos, naturais, educacionais, entre outros. Logo, a obesidade exógena (ocasionada por hábitos alimentares inadequados e sedentarismo) é mais predominante entre os estudos quando comparada a obesidade endógena (ocasionada por síndromes genéticas). Para estes mesmos autores, existem hábitos errôneos no cotidiano das crianças, como o sedentarismo e alimentação com excesso de calorias, que exercem influência na massa corpórea, fazendo com que estas crianças venham a desenvolver obesidade na vida adulta (MENDES JOH, et al., 2019).

O crescente número de crianças com obesidade deve ser levado em consideração pelo fato de vir acompanhado por comorbidades na vida adulta. Logo, faz-se necessário diagnosticar e tratar essa patologia o quanto antes, através da verificação do perfil genético familiar e dos fatores de risco para o desenvolvimento da obesidade; configurando um plano que tenha como objetivo diminuir o risco para aparecimento de futuras comorbidades (LINHARES FMM, et al., 2016).

A falta de uma dieta balanceada, estimula o consumo exagerado de alimentos gordurosos e calóricos, prejudicando a qualidade de vida da criança. Esse hábito alimentar não saudável é decorrente da correria do dia a dia e apresenta como consequência o preparo dos alimentos de uma forma mais rápida e prática, além de incentivar o consumo de alimentos industrializados; estes alimentos por sua vez, afetam de forma gradativa o funcionamento corporal. Além da rotina agitada, deve-se adicionar como fator predisponente para obesidade o sedentarismo, que vem acompanhado do uso excessivo de aparelhos celulares e redes sociais (OLIVEIRA LC, et al., 2017; LIRA AK, et al., 2020).

A família tem papel fundamental no estilo de vida que será adotado pela criança, principalmente os pais, logo cabe a estes disponibilizar alimentos nutritivos e condições de se praticar atividades físicas, visto que a infância é a fase mais importante para a formação dos hábitos que a criança terá por toda vida. De modo geral, as recentes pesquisas mostram que pais autoritários têm filhos com padrão de normalidade relacionado a peso e IMC, enquanto pais permissivos são os que encontram filhos com maiores alterações nesses mesmos padrões (DORNELLES AD, et al., 2014).

Esse papel de influência exercido pelo núcleo familiar interage diretamente com os demais ambientes em que a criança está inserida, como colégio; logo pode-se afirmar que a criança é totalmente subordinada do ambiente onde vive, assim seus hábitos são constantemente reflexo destes meios. Cabe a família então, promover comportamentos, estilo de vida e hábitos alimentares mais saudáveis (MENDES JOH, et al., 2019).

O estudo de Miranda JMQ, et al. (2015) mostrou que o sobrepeso e obesidade infantil podem estar associados ao fator socioeconômico, levando a um estado desfavorável de saúde, podendo favorecer precocemente o desenvolvimento de doenças crônicas. O IMC é um índice importante para diagnosticar o estado nutricional de crianças, que se correlaciona com o percentual de gordura. $O$ desenvolvimento de estratégias e intervenção é útil para prevenir a obesidade infantil.

O estudo transversal de Almeida NB, et al. (2020), realiza uma análise da influência familiar na obesidade infantil: interrupção do aleitamento materno exclusivo de maneira precoce. Segundo a pesquisa realizada, das 366 crianças elegíveis no estudo apenas $6 \%$ receberam aleitamento materno exclusivo, o que explica a introdução de alimentos errôneos de forma precoce, justificando o excesso de peso nessas crianças.

Além disso, estudos de Bertoletti J e Garcia-Santos SCG (2012) expõem que 100\% das crianças dispõem de pelo menos um familiar obeso ou com sobrepeso, podendo ser desde pais até avós; e que além da influência na alimentação, o fator genético transmitido a cada geração intervém diretamente na criança, podendo predispor uma futura obesidade. Além da genética, a presença de indivíduos obesos no ambiente familiar, pode proporcionar uma aprendizagem de hábitos alimentares não saudáveis, a partir da identificação com os mesmos (LINHARES FMM, et al., 2016; MENDES JOH, et al., 2019).

Segundo, Eskenazi EMS, et al. (2018) e Pazin DC, et al. (2017) o primeiro indício para uma futura obesidade é o sobrepeso na infância, logo não deve ser um fator tratado de forma negligente. Diante deste 
contexto, é notório que a obesidade infantil é algo grave, que pode levar a possíveis consequências na vida adulta, como aumento da pressão arterial, desenvolvimento de Diabetes Mellitus e cardiopatia (LIRA AK, et al. 2020).

Os alimentos ultraprocessados e os açúcares são os principais malefícios que ajudam a obesidade infantil a perdurar na vida da criança, o meio de convívio social (escola) é o principal vínculo deste tipo de alimento. Por isso é importante que os gestores escolares, os professores e a família conscientizem as crianças dos benefícios da alimentação saudável, como também fornecer no ambiente escolar o alimento correto e saudável. Proibindo a venda e entrada de alimentos açucarados e ultraprocessados, são meios preventivos e educativos de minimizar os casos de obesidade infantil (ESKENAZI EMS, et al., 2018).

Em razão da grande correria cotidiana dos adultos, onde o tempo é pouco, os alimentos são preparados de forma rápida, principalmente alimentos industrializados prontos para o consumo rápido e prático, esses são os piores, e que afetam de forma gradativa o mau funcionamento do corpo, deixando a criança mal alimentada e com o uso contínuo de celulares e redes sociais, fez com que muitos filhos ficassem sedentários, com isso os casos de obesidade infantil só tendem ao crescimento gradativo (OLIVEIRA LC, et al., 2017).

Além das complicações supracitadas, considerando o cenário atual, deve ser levado em consideração que a obesidade não é só causadora de algumas patologias, mas pode ser considerada também fator agravante de outras infecções, como a do SARS-CoV-2 (Novo Corona Vírus). Por causar um processo inflamatório crônico no organismo, a obesidade altera o funcionamento do sistema imunológico, levando a um agravamento do quadro de COVID-19 em crianças (COSTA LR, et al., 2020).

Devemos levar em consideração não só os problemas na saúde física do paciente, mas também os problemas psicológicos causados pela obesidade. A pessoa obesa, tem maiores chances de passar por situações de intimidações quando são tratados com desdém, repugnância e aversão, levando a uma visão negativa do próprio corpo. Além disso, podem ser julgados por não solucionarem o problema do sobrepeso e ainda repreendidos pela disciplina deficiente; dentre os principais agentes instigadores estão os profissionais de saúde, principalmente os especializados em estética e dieta. A soma de todos esses fatores leva a presença de sentimento de rejeição no indivíduo obeso, desenvolvendo até mesmo casos de depressão. A falta de perspectiva em conjunto com sentimento de vergonha, são somados ao histórico psicológico deste paciente (MENDES JOH, et al., 2019).

No estudo qualitativo realizado por Moraes PM e Dias MCSB (2013); fica evidente a influência familiar na autoestima das crianças obesas; segundo esse estudo, a autoestima é fruto dos diferentes relacionamentos vivido pelas crianças ao longo dos anos, nos diferentes ambientes em que estão inseridas. Além da baixa autoestima, outro fator prevalente observado nesses mesmos indivíduos é a ansiedade, bem como quadros depressivos, podendo levar ao aparecimento de crianças cada vez mais agressivas e nervosas (MENDES $\mathrm{JOH}$, et al., 2019).

Para Fechine ADL, et al. (2017); o Brasil passa por uma mudança extremamente radical, a qual os numerosos casos de desnutrição infantil vêm sendo substituídos por aumento no número de crianças obesas ou com sobrepeso; alterando assim as preocupações do governo em relação ao quadro de saúde da população (LIRA AK, et al., 2020).

Importante salientar os fatores que vêm alterando a estrutura familiar, levando a mesma ao sedentarismo, uma vez que a influência da família é o principal modulador da obesidade na infância. Com o desenvolvimento da tecnologia, surgiram aparelhos como: televisões que se conectam à internet, computadores, videogames e Smartphones que vêm cada vez mais fazendo parte da rotina familiar. Esses avanços levam ao comodismo do indivíduo, que não precisa se deslocar para realização de atividades; resolvendo os assuntos necessários com apenas um "clique" (LOPES PCS, et al., 2010).

Além de proporcionar o comodismo por facilitar as tarefas diárias, as novas tecnologias vêm substituindo também as atividades recreativas infantis. Logo, atividades que exigiam esforços físicos como jogar bola e pular corda, por exemplo, foram trocadas por jogos online, no qual não há o mesmo gasto energético, facilitando o surgimento do sobrepeso (LOPES PCS, et al., 2010). 
O conhecimento dos efeitos benéficos do exercício físico no tratamento e na prevenção da obesidade infantil nos mostra a relevância dos profissionais da área da saúde adotarem estratégias que estimulem as crianças a praticarem regularmente atividade física. Ressalta-se a importância das aulas de educação física como uma forma de manutenção e incentivo para alcançar um estilo de vida ativo nas crianças em idade escolar (TESTA WL, et al., 2017).

Em razão de todos os contextos que foram analisados, foi notado grande necessidade de estudos voltados para os programas nutricionais fornecidos pelo governo, como: Política Nacional de Alimentação e Nutrição (PNAN), Promoção da Alimentação Adequada Saudável (PAAS), Políticas Nacionais de Promoção da Saúde (PNPS), Rede de Atenção à Saúde (RAS), Programa Saúde na Escola (PSE), Política Nacional de Segurança Alimentar e Nutricional (PNSAN), Programa de Aquisição de Alimentos (PAA), Programa Nacional de Alimentação Escolar (PNAE), Segurança Alimentar Nutricional (SAN) (HENRIQUES P, et al., 2018).

Percebe-se a obesidade como uma epidemia em crianças, devendo ser tratada como saúde pública, necessita de apoio familiar, sendo o principal ponto de apoio alicerce na mudança de hábitos alimentares, propiciando a criança a se tornar um adulto saudável futuramente. Cabe a família cuidar da alimentação de seus filhos, ensinando-lhes o que é ou não prejudicial a saúde deles. Destaca=se que a obesidade é decorrente de uma série de fatores, os principais são o consumo exagerado de alimentos não saudáveis, tais como: alimentos açucarados e ultra processados, associados ao sedentarismo e também multifatorial, que são fatores ambientais e genéticos, que podem associar-se ao diagnóstico. A alimentação adequada e a prática de exercícios físicos regulares podem melhorar a qualidade de vida das crianças, pois a obesidade acarreta diversas adversidades à saúde (LIRA AK, et al, 2020).

\section{CONSIDERAÇÕES FINAIS}

O contexto onde a criança está inserida, tanto familiar, o escolar quanto o social, é um componente facilitador para manifestação de obesidade na infância, que por sua vez influencia no desenvolvimento de diversas patologias na vida adulta. O sobrepeso e a obesidade infantil contribuem não só para surgimento de patologias físicas na vida adulta, mas também para patologias psicológicas, abordadas na grande maioria dos artigos aqui revisados. Apesar das limitações impostas pelo atual padrão econômico que incentiva o consumo de alimentos industrializados, é necessário a promoção e prevenção da obesidade infantil, com intuito de desenvolver hábitos alimentares mais saudáveis, objetivando alterar os comportamentos sedentários e patogênicos que se relacionam entre si.

\section{REFERÊNCIAS}

1. ALMEIDA NB, et al. Pre and perinatal factors associated with weight gain among preschool children enrolled at day care centers. Rev. paul. pediatr., 2020; 38.

2. ANDRADE RS, et al. Obesidade e dislipidemia na infância: uma revisão sobre associação de marcadores laboratoriais. Rev.bras.anal.clin (RBAC), 2018; 50(3): 207-214.

3. ANDRADE TM, et al. Problemas Psicológicos e Psicodinâmicos de crianças e adolescentes obesos: relato de pesquisa. Psicol. cienc. prof, 2014; 34(1): 126-141.

4. BERTOLETTI J, SANTOS SCG. Avaliação do Estresse na Obesidade Infantil. PSICO, PUCRS, 2012; $43(1)$ : $32-38$.

5. CAMARGO APPM, et al. A não percepção da obesidade pode ser um obstáculo no papel das mães de cuidar de seus filhos. Ciênc. saúde coletiva, 2013; 18(2: 323-333.

6. COSTA LR, et al. Obesidade infantil e quarentena: crianças obesas possuem maior risco para a COVID-19. Residência Pediátrica, 2020; 10(2): 1-6.

7. COUTINHO VM, et al. Estilo de apego em crianças com doenças crônicas: uma revisão integrativa. Rev. paul. pediatr., 2020; 38.

8. DORNELLES AD, et al. O papel da sociedade e da família na assistência ao sobrepeso e à obesidade infantil: percepção de trabalhadores da saúde em diferentes níveis de atenção. Saúde soc, 2014; 23(4): 1275-1287.

9. HENRIQUES P, et al. Políticas de Saúde e de Segurança Alimentar e Nutricional: desafios para o controle da obesidade infantil. Ciênc. saúde coletiva, 2018; 23(12): 4143-4152.

10. LINHARES FMM, et al. Obesidade infantil: influência dos pais sobre a alimentação e estilo de vida dos filhos. Temas em Saúde, 2016; 16(2): 460-481. 
11. LIRA AK, et al. Determinantes da obesidade infantil: um problema de saúde pública. Temas em Saúde, 2020; 20(1): 77-94.

12. LOPES PCS, et al. Fatores de risco associados à obesidade e sobrepeso em crianças em idade escolar. Rev. bras. enferm., 2010; 63(1): 73-78.

13. MENDES JOH, et al. Características psicológicas e relações familiares na obesidade infantil: uma revisão sistemática. Rev. SBPH, 2019; 22(2): 228-247.

14. MIRANDA JMQ, et al. Prevalência de sobrepeso e obesidade infantil em instituições de ensino: públicas vs. privadas. Rev Bras Med Esporte, 2015; 21(2): 104-107.

15. OLIVEIRA LC, et al. Excesso de peso, obesidade, passos e atividade física de moderada a vigorosa em crianças. Rev Saúde Pública, 2017; 51(38): 1-12.

16. PAIVA ACT, et al. Obesidade Infantil: análises antropométricas, bioquímicas, alimentares e estilo de vida. Revista Cuidarte, 2018; 9(3): 2387-99.

17. PÉREZ LM, MATTIELLO R. Determinante da composição corporal em crianças e adolescentes. Revista Cuidarte, PUCRS, 2018; 9(2): 2093-104.

18. SANTOS LRC, RABINOVICH EP. Situações familiares na obesidade exógena infantil do filho único. Saude soc., 2011; 20(2): 507-521.

19. TASSARA V, et al. Importância do contexto sociofamiliar na abordagem de crianças obesas. Rev. paul. pediatr., 2010; 28(3): 309-314.

20. TESTA WL, et al. Exercício físico com atividades recreativas: uma alternativa para o tratamento da obesidade. Revista Brasileira de Obesidade, Nutrição e Emagrecimento (RBONE), 2017; 11 (62): 49-55. 